\title{
Acute suppurative thyroiditis with thyroid metastasis from oesophageal cancer
}

\author{
Le Dai, Sitong Lin, Dianyuan Liu, Qing Wang \\ China-Japan Union Hospital of Jilin University, Department of Endocrinology, Jilin, China
}

Key words: suppurative thyroiditis; thyroid neoplasms; neoplasm metastasis; oesophageal neoplasms

\section{Introduction}

Acute suppurative thyroiditis (AST), accounting for $0.1-0.7 \%$ of all thyroid diseases, is a rare infectious disease. The incidence of metastatic spread of malignancies to thyroid is also relatively low at about $1.4-3.0 \%$ of all thyroid malignant tumours. Thyroid metastasis originating from the oesophagus is poorly documented. Herein, we report the first case of AST complicated with thyroid metastasis from oesophageal cancer.

\section{Case presentation}

A 66-year-old male presented to our hospital with a one-month history of fever, neck pain and swelling. A review of systems was negative for other symptoms. He had no other medical history except for diabetes. He did not take any medication before presentation. His axillary temperature was $37.6^{\circ} \mathrm{C}$, heart rate was 96 beats $/ \mathrm{min}$, and blood pressure was 105/70 mm Hg. Physical examinations showed diffuse enlargement of thyroid with tenderness to palpation, predominantly the left lobe. A nodule could be touched in the left lobe. His thyroid function tests showed a decreased thyroid-stimulating hormone concentration of $0.005 \mathrm{mIU} / \mathrm{L}(0.372-4.94)$, increased free $\mathrm{FT}_{3}-9.85$ $\mathrm{pmol} / \mathrm{L}(2.1-6.80)$, and $\mathrm{FT}_{4}-62.2 \mathrm{pmol} / \mathrm{L}(12.0-22.0)$. He did not have any thyroid antibodies. The patient was admitted to the Endocrinology Department for further examinations and treatment.

Laboratory workup showed an elevated WBC count of $22.60 \times 10^{9} / \mathrm{L}$ (4-10) with differential showing $88.4 \%$ (50-70) neutrophils. CRP was $66.04 \mathrm{mg} / \mathrm{L}(0-5)$ and ESR was $44 \mathrm{~mm} / \mathrm{h}(0-15)$. Syphilis and HIV antibody were negative. Blood culture was negative for fungi, acid-fast bacilli, anaerobes, and aerobes. Initial ultrasonography of the thyroid revealed an enlarged thyroid with heterogeneous texture and multiple nodules that were well-circumscribed, suggesting multinodal goitre. Notably, a heterogeneous and mixed echoic lesion with ill-defined margins, irregular form, and little liquefactive region was observed in the left lower lobe and isthmus, suggesting an inflammatory mass. It measured approximately $4.76 \times 3.61 \mathrm{~cm}$. Plain CT of the thyroid provided no further information regarding the inside of the nodules. Iodine-131 scan revealed a large photon deficient area in the thyroid.

The suspicion of AST was raised by examinations. Although no radio graphic evidence of abscess was noted, antibiotics were initiated. The patient was started on cefoperazone, and his blood glucose was well controlled. Repeated ultrasonography of thyroid was performed to monitor changes of the mass. A magnetic resonance imaging of thyroid was performed on day 9 , which confirmed a thyroid abscess in the left lower lobe and isthmus. The patient subsequently underwent an ultrasound-guided paracentesis and drainage of the abscess. Findings on cytological examination were suggestive of AST because no atypical wells were evident, and inflammatory cells were seen in the background. AST was diagnosed. The aspirate culture grew staphylococcus. Although the patient was transitioned from cefoperazone to meropenem and vancomycin during hospitalisation, the antibiotic therapy produced no response. His symptoms worsened, WBC and neutrophils improved, and the abscess did not get smaller. We considered the presence of malignant tumours. An ultrasound-guided FNA targeting the hypoechoic lesion in the left lower lobe was performed. The pathology report of aspirate smears showed atypical cells, which were negative for TTF-1 and Tg, and positive for CK5, P63, P40, and CK7, supporting squamous cell carcinoma. PET/CT was performed to assess primary and metastatic carcinoma. It showed the middle part of the 
oesophagus with increased glucose metabolism, considered to be primary malignant tumour, and suprasternal fossa with increased glucose metabolism adjacent to the left lobe of the thyroid, considered to be metastatic malignant tumour. It also showed mediastinal lymph node metastases. Thyroid metastasis from oesophageal cancer was diagnosed. We suggested that the patient undergo further examinations to develop treatment. However, he refused and requested to be discharged.

\section{Discussion}

In general, resistance of thyroid to invading organisms is thought to be due to complete encapsulation, generous vascular supply and lymphatic drainage, and the presence of iodine locally. The metastatic spread of malignancies to the thyroid rarely happens because of the lack of filtering capability or rapid blood flow of the thyroid inhibiting the contact between tumour cells and thyroid. In this case, the patient was an elderly diabetic patient with a low state of immunocompetence. He suffered AST and multinodal goitre, and the pathological changes had destroyed the anatomical and physiological features of thyroid, which may lead to the occurrence of thyroid metastasis.

When an abscess is not formed, the clinical pictures of AST are similar to subacute thyroiditis (SAT), which is easily misdiagnosed. However, the treatments for these two diseases are totally different. Previous studies have reported that the symptoms of patients with AST worsen after glucocorticoid therapy. The case prompts physicians to consider that the diagnosis of AST should be based on high clinical suspicion, especially for patients whose imaging examinations are unclear. We should be wary of AST when patients have the following doubtful points: 1 - severe systemic inflammatory response complicated with significantly increased WBC and neutrophils; 2 - left lobe involvement; 3 - ultrasound characterised by mixed echoic lesion with liquefactive necrosis; and 4 - thyroid function is normal, few present with hyperthyroidism or hypothyroidism [2]. This patient was suspected of SAT. We avoided prescribing glucocorticoids and started antibiotic therapy for the first time.

Generally, AST produces complete response and recovers entirely after reasonable antibiotic therapy, even in cases that AST complicated with primary thyroid malignant cancer $[3,4]$. However, this case was uncommon in terms of treatment effect and prognosis of disease. On the one hand, the existent AST leaded to thyroid metastasis, and metastatic carcinoma facilitated suppurative infection. On the other hand, the patient was diagnosed at the terminal stage of oesophageal cancer, and his general status was poor; these two factors led to ineffective treatment. Thyroid metastasis is easily misdiagnosed due to the concealed onset of thyroid metastasis. It often presents with thyroid mass as the initial symptom and is regarded as terminal expression and poor prognosis of malignant tumours 5 . Physicians should be alert to thyroid metastasis when engaging with patients who present with thyroid mass.

\section{Conclusion}

When a patient presents to a hospital because of fever, neck pain, and swelling, AST should be considered. The diagnosis of AST should be based on high clinical suspicion. For those who are highly suspected of AST, glucocorticoids should be avoided, and antibiotic therapy should be prescribed as soon as possible. In addition, thyroid cancer should be taken into account, especially when antibiotic therapy is ineffective. Notably, except for primary thyroid cancer, thyroid metastasis should be considered. This is the first case of AST complicated with thyroid metastasis from oesophageal cancer, and it throws new light on AST and thyroid metastasis for physicians.

\section{Disclosure statement}

The author declares no conflicts of interest.

\section{Funding}

The author has not declared a specific grant for this research from any funding agency in the public, commercial, or not-for-profit sectors.

\section{Competing interests}

None declared.

\section{References}

1. Yegya-Raman N, Copeland T, Parikh P. Acute Suppurative Thyroiditis in an Intravenous Drug User with a Preexisting Goiter. Case Rep Med. 2018, 2018: 5098712, doi: 10.1155/2018/5098712, indexed in Pubmed: 29780423.

2. Spitzer M, Alexanian S, Farwell AP. Thyrotoxicosis with post-treatment hypothyroidism in a patient with acute suppurative thyroiditis due to porphyromonas. Thyroid. 2012; 22(1): 97-100, doi: 10.1089/thy.2011.0159, indexed in Pubmed: 22136210.

3. Otani H, Notsu M, Koike S, et al. Acute suppurative thyroiditis caused by thyroid papillary carcinoma in the right thyroid lobe of a healthy woman. Thyroid Res. 2018; 11: 4, doi: 10.1186/s13044-018-0049-6, indexed in Pubmed: 29785209.

4. Kalladi Puthanpurayil S, Francis GL, Kraft AO, et al. Papillary thyroid carcinoma presenting as acute suppurative thyroiditis: A case report and review of the literature. Int J Pediatr Otorhinolaryngol. 2018; 105: 12-15, doi: 10.1016/j.ijporl.2017.11.032, indexed in Pubmed: 29447798.

5. Zhao Y, Tian Z, Huang Y. Clinical analysis of four cases of thyroid metastasis. Chin J Cancer Prev Treat. 2018; 25: 295-297. 